Original

Article

\title{
Intermediate-term Results after Surgery for Anomalous Origin of the Left Coronary Artery from the Pulmonary Artery
}

\author{
Ahmet Kirbas, MD, Onur Gurer, MD, and Mehmet Salih Bilal, MD
}

\begin{abstract}
Purpose: Anomalous origin of the left coronary artery from the pulmonary artery is optimally treated by creating a dual coronary system. Our aim was to review the results of operations performed in these patients and determine the intermediate-term outcomes for left ventricular function and mitral regurgitation.

Methods: Between July 2004 and January 2009 seven patients (5 boys, 2 girls) aged between 4 months and 12 years (median, 4.5 years) were operated for anomalous origin of the left coronary artery from the pulmonary artery. The surgical correction was either performed by direct implantation (58\%) or restoration of a composite tunnel (42\%). Simultaneous mitral annuloplasty was performed in one patient with severe mitral regurgitation and simultaneous total correction of tetralogy of Fallot was performed in another.

Results: There was no hospital or late deaths. Postoperative echocardiograms demonstrated a significant improvement in the left ventricular ejection fraction $(52 \% \pm 6 \%$ versus $39 \% \pm 8 \%$, $P=0.02)$ and mitral regurgitation $(11 \%$ mild versus $48 \%$ moderate, $P=0.02)$ compared to those obtained preoperatively.

Conclusion: Direct re implantation of the left coronary artery to the aorta and restoration of a composite tunnel from aortic and pulmonary artery walls are equally effective techniques with an acceptable operative mortality, excellent cardiac recovery, and intermediate survival.
\end{abstract}

Keywords: congenital cardiac disease, echocardiography, mitral valve, mitral regurgitation, left ventricular function, pulmonary artery, left coronary artery

\section{Introduction}

Anomalous origin of the left coronary artery from the pulmonary artery is a rare congenital anomaly which was first described by Brooks in 1886. ${ }^{1}$ It is usually seen as an isolated lesion. ${ }^{2)}$ The incidence is 1 in 300000 live births, and it affects $0.25 \%$ to $0.5 \%$ of children with con-

$\overline{\text { Department of Cardiovascular Surgery, Medicana Hospitals }}$ Camlica, Istanbul, Turkey

Received: March 22, 2011; Accepted: July 12, 2011

Corresponding author: Onur Gurer, MD. Medicana Camlica Hastanesi, Alemdag Cad.No:85 Uskudar Istanbul, 34764, Turkey

Email: onurgurermd@yahoo.com

(C)2011 The Editorial Committee of Annals of Thoracic and Cardiovascular Surgery. All rights reserved. genital heart disease. ${ }^{3)}$ It is one of the most common causes of myocardial ischemia and infarction in children, and if not treated, results in mortality rate of up to $90 \%$ within the first year of life. ${ }^{4)}$ During the neonatal period, increased pulmonary artery pressures, resulting from the high pulmonary vascular resistance, lead to an antegrade flow from the pulmonary artery into the anomalous left coronary artery. However, as the pulmonary vascular resistance gradually decreases, left-to-right shunting increases. Consequently, left ventricular perfusion becomes dependent on intracoronary collateral circulation from an enlarged right coronary artery. Coronary steal results in ischemia, and subsequent infarction of left ventricular myocardium and anterolateral papillary muscle ischemia leads to mitral regurgitation. The current standard management in patients with anomalous origin of the left 
coronary artery from the pulmonary artery is immediate surgical correction on diagnosis, by restoration of a twocoronary artery system. ${ }^{5)}$ Different techniques have been described such as ligation of anomalous left coronary artery, creation of an intrapulmonary tunnel with an aortopulmonary window, creation of an extrapulmonary composite tunnel, and direct implantation. Our surgical technique has evolved with time, and in cases in which anomalous left coronary artery is distant to the aorta, we use previously described implantation techniques to facilitate a tension-free transfer of the coronary artery to the aorta. Herein, we report our experience with two different techniques and assess postoperative cardiac recovery of cardiac function and intermediate-term clinical outcomes.

\section{Material and Methods}

The surgical database of our hospital was retrospectively reviewed for surgical correction cases performed for anomalous origin of the left coronary artery from the pulmonary artery, between July 2004 and January 2009. Clinical, operative, and outcome data of the patients were recorded.

Between July 2004 and January 2009, 7 patients with anomalous origin of the left coronary artery from the pulmonary artery were operated on at our hospital using direct implantation (57\%) or the composite tunnel technique $(42 \%)$. All patients were operated on at the time of presentation. Approval of this study was obtained from the Research Ethics Board at our hospital.

\section{Surgical technique}

The operation was performed through a median sternotomy. The pericardium was opened to the left of the midline and an autologous pericardial patch was obtained for later use. The patch was fixed in glutaraldehyde afterwards. High aortic cannulation, brachiocephalic vein cannulation and cannulation of the inferior caval vein was performed. The left ventricle was decompressed by venting through the right superior pulmonary vein or interatrial septum. With the use of moderate hypothermia, antegrade cardioplegia ( $30 \mathrm{ml} / \mathrm{kg}$ body weight) was administered into the ascending aorta. Once cardiopulmonary bypass was initiated, the left and right pulmonary arteries were snared to prevent myocardial ischemia secondary to steal of coronary blood flow into the pulmonary artery. The aorta was cross-clamped and the heart arrested with antegrade cardioplegia (30 ml/kg body weight) infused into the ascending aorta, and the main pulmonary artery was opened horizontally.

We used two different techniques in our patients. If the anomalous left coronary artery originated from the posterior pulmonary sinus, the coronary ostium was excised with a wide cuff of pulmonary arterial wall to form a coronary button. The coronary button was widely mobilized to allow a tension-free anastomosis. Similarly, an incision was made at the posteromedial wall of the aorta for the anastomosis. The resultant defect in the pulmonary artery was patched with autologous pericardium. We used a modified technique that has been described previously by Sese and colleagues ${ }^{6}$ that combined the aortic and pulmonary flaps, in cases in which the anomalous coronary artery arose from the nonfacing pulmonary sinus or from the posterior pulmonary sinus close to the commissure with the nonfacing sinus. The orifice of the anomalous origin of the left coronary artery from the pulmonary artery was carefully identified in the nonfacing sinus of the pulmonary artery. As the artery arose at the farthest point from the ascending aorta, direct implantation into the ascending aorta was technically impossible. The coronary button was taken along with a flap of pulmonary artery wall transversely toward the anterior aspect, above the valve commissure to make the superior wall of the composite tunnel. Similarly, a flap of the aortic wall was prepared to produce the inferior wall of the composite tunnel (Fig. 1).

\section{Postoperative management}

Early extubation was performed in all patients who met the required criteria. Discontinuation of inotropic drugs and intravenous vasodilators, which was determined by bedside transthoracic echocardiographic assessment of ventricular function and degree of mitral regurgitation, was accomplished as quickly as the clinical status allowed. All patients were extubated within 24 hours and inotropes were discontinued within 36 hours, except for one patient (a neonate), who was extubated on day 2 and had inotropes discontinued on day 3. Discharge echocardiograms were performed in all patients. The patients were all discharged on aspirin $5 \mathrm{ml} / \mathrm{kg}$ per day for 3 months.

\section{Statistical analysis}

Data were presented as mean plus or minus standard deviation and median (range) where appropriate. Comparison of preoperative and postoperative variables was performed using the Student $t$-test; $P$ values were considered significant if smaller than 0.05 . 


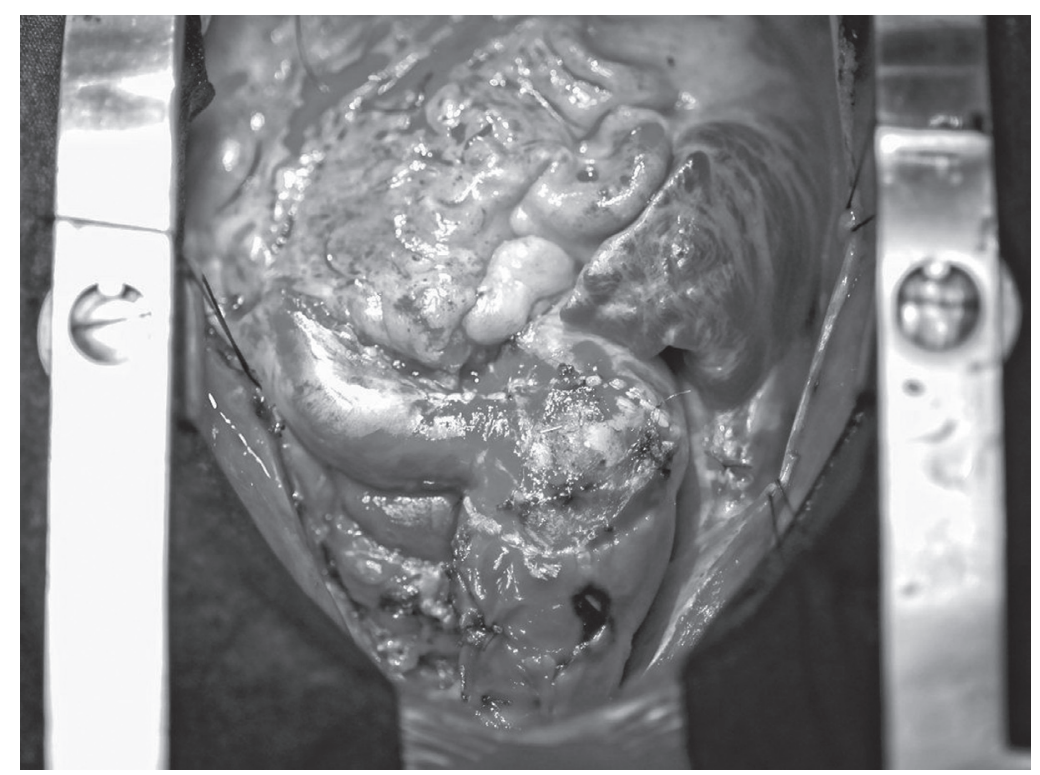

Fig. 1 The new coronary artery was successfully implanted into the ascending aorta using the composite tunnel technique.

\section{Results}

\section{Patient characteristics}

Seven patients, 5 boys and 2 girls; aged between 4 months and 12 years (median, 4.5 years) had surgical corrections for anomalous origin of the left coronary artery from the pulmonary artery between July 2004 and January 2009. The body weight ranged from 4.2 to 31.0 $\mathrm{kg}$ (median, $14.8 \mathrm{~kg}$ ). Except for one patient who presented with heart failure and severely impaired left ventricular function, none of the patients had evidence of heart failure. Chest roentgenography revealed cardiomegaly, with a cardiothoracic ratio between 0.6 and 0.72 (mean, $0.68 \pm 0.17$ ). The electrocardiograms of the patients revealed pathologic $\mathrm{Q}$ waves in the anterolateral leads. The preoperative diagnosis was established by twodimensional echocardiography and Doppler studies. The site of the anomalous origin of the coronary artery from the pulmonary artery was the right sinus of Valsalva in three patients, the left sinus of Valsalva in two patients, and the far left sinus in two patients. The diagnosis was confirmed by cardiac catheterization and aortography (Table 1).

There were no early deaths or late deaths during the follow-up period of 12 to 42 months (Table 2). The average length of stay in the intensive care unit was $2.6 \pm 1.1$ days. The median duration of intensive care treatment was 8 days (range, 1-24 days). Complications encoun-
Table 1 Preoperative clinical symptoms and findings in children with anomalous origin of the left coronary artery from the pulmonary artery (seven patients)

\begin{tabular}{lc}
\hline Symptoms & Incidence $(\%)$ \\
\hline Poor exercise tolerance & $5(71 \%)$ \\
Poor weight gain & $3(42 \%)$ \\
Tachypnea & $3(42 \%)$ \\
Chest pain & $2(28 \%)$ \\
Anxiety & $1(14 \%)$ \\
Suspected respiratory infection & $1(14 \%)$ \\
Preoperative findings & \\
Electrocardiogram abnormalities & $7(100 \%)$ \\
Myocardial infarction/ischemia & $3(42 \%)$ \\
Left atrial enlargement & $2(28 \%)$ \\
Left ventricular hypertrophy & $3(42 \%)$ \\
Mitral regurgitation & $6(85 \%)$ \\
Mitral prolapse & $2(28 \%)$ \\
Catheterization & \\
Performed & $7(100 \%)$ \\
\hline
\end{tabular}

tered during the early postoperative period were; rhythm disorder in one patient, neurologic disorder in one patient and the need for pericardial drainage in another patient. Postoperative echocardiographic examinations were available for all patients and angiographic evaluation could be performed in two patients. Patency and prograde flow of the new coronary artery system was demonstrated in each case, and no pulmonary artery obstructions were evident (Fig. 2). No new angina occurred and 
Table 2 Characteristics of the patients with anomalous origin of the left coronary artery from the pulmonary artery

\begin{tabular}{|c|c|c|c|c|c|}
\hline Patient no & Year of diagnosis & Age (years) & Weight(kilogram) & Procedure & $\begin{array}{c}\text { Follow-up } \\
\text { (months) }\end{array}$ \\
\hline 1 & 2000 & 0.31 & 4.5 & Direct implantation & 42 \\
\hline 2 & 2005 & 1.75 & 11 & Direct implantation & 36 \\
\hline 3 & 2006 & 0.29 & 4.2 & $\begin{array}{l}\text { Direct implantation - } \\
\text { Total correction of TOF }\end{array}$ & 34 \\
\hline 4 & 2007 & 11 & 24 & Composite Tunnel & 21 \\
\hline 5 & 2008 & 12 & 31 & Composite Tunnel & 17 \\
\hline 6 & 2008 & 0.75 & 6.5 & $\begin{array}{l}\text { Composite Tunnel - } \\
\text { Mitral valve plasty }\end{array}$ & 15 \\
\hline 7 & 2009 & 6 & 23 & Direct implantation & 12 \\
\hline
\end{tabular}

TOF: tetralogy of fallot

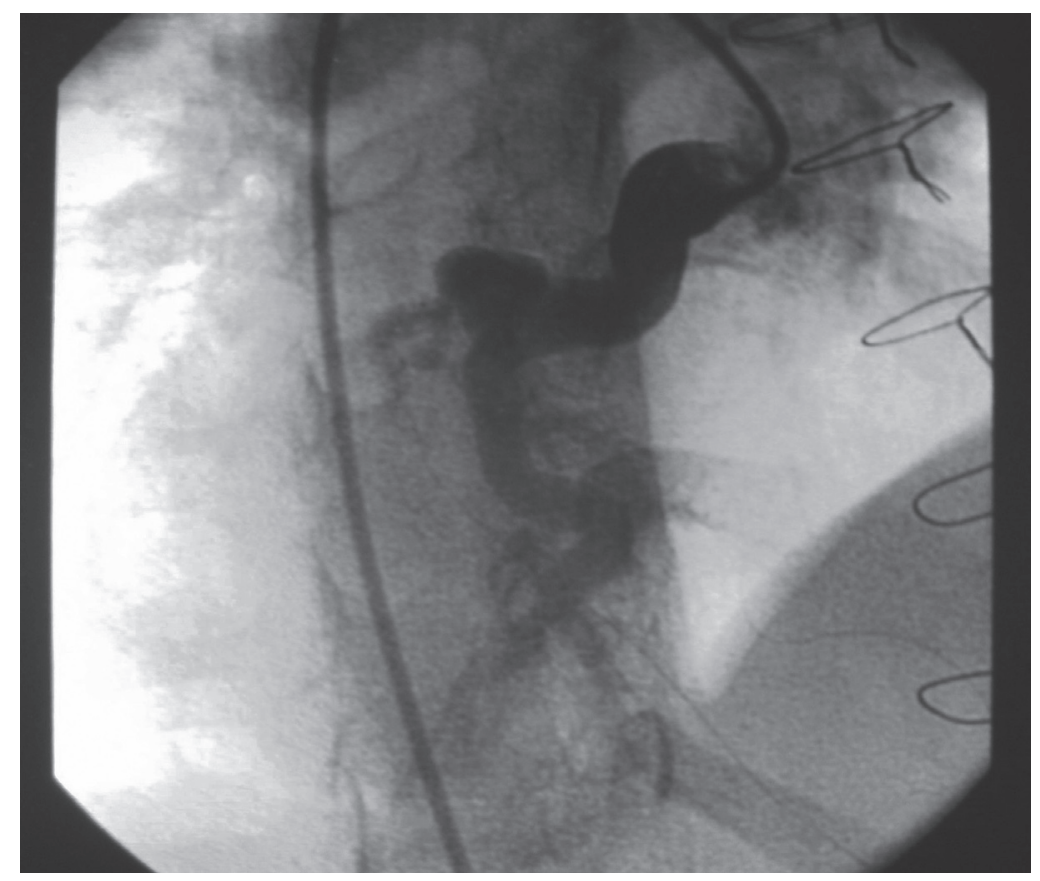

Fig. 2 Ascending aortogram taken twelve months after repair of anomalous origin of the left coronary artery from the pulmonary artery using a composite extrapulmonary tunnel shows excellent flow in the coronary artery.

no new infarctions were demonstrated on electrocardiography.

\section{Echocardiographic evaluations}

\section{Determination of left ventricular ejection fraction and right ventricular outflow tract obstruction}

Left ventricular function improved in six of the seven patients, and the improvement was significant $(P=0.02)$. The mean preoperative left ventricular ejection fraction improved from $39 \% \pm 8 \%$ to $52 \% \pm 6 \%$ after the surgery. None of the patients had residual right ventricular outflow tract obstruction on Doppler echocardiography at discharge. At 12- months follow-up, left ventricular ejection fraction was normalized in all patients $(61 \% \pm 3 \%)$ and remained so on subsequent follow-up examinations.

\section{Mitral valve function}

All except one patient had mitral regurgitation; mitral regurgitation was severe in one patient, moderate in three patients and mild in two patients, Mitral valve prolapse was present in two patients.

Posterior annuloplasty using 4/0 polydioxone suture was performed in only one patient who had severe mitral 
regurgitation. Residual mitral regurgitation in this patient was grade two. The residual mitral regurgitation of the other patients at discharge was graded as 0 (four patients), I-II (one patient) and II-III (one patient). No residual mitral regurgitation was detected at eight months postoperatively.

\section{Follow-up and data analysis}

Patients were re-evaluated at three and six months and annually thereafter. Echocardiographic assessments included evaluation of left ventricular ejection fraction and mitral regurgitation.

\section{Discussion}

Anomalous origin of the left main coronary artery from the pulmonary artery is a rare disorder making-up about $0.25 \%$ of children with congenital heart disease. ${ }^{7)}$ Signs and symptoms of the disease mostly develop in the first few months of life and a great majority of the infants die within the third and sixth months of life. Attempts to resolve the ischemia from anomalous origin of the left main coronary artery from the pulmonary artery have evolved out of palliative procedures, and techniques including providing bypasses using various types of conduits, intrapulmonary tunneling, and direct coronary translocation have been used. ${ }^{7)}$ The combined operative mortality of these restorations, that do not result in a twocoronary system, have a high mortality $(0 \%-23 \%)$ and continued poor ventricular function. ${ }^{8)}$

There are two essential principles in the surgical management of anomalous origin of the left main coronary artery from the pulmonary artery. The first one is the need for successful restoration of a two-vessel coronary blood supply to achieve the lasting recovery of the myocardium, and to decrease the incidence of late sudden death. ${ }^{9)}$ Additionally, the procedure should provide longterm patency of the reconstructed coronary artery and allow for growth and enlargement with age.

Several techniques to avoid any tension on the implanted anomalous coronary artery have been described in the literature. In order to lengthen the implanted coronary artery, Sese and colleagues ${ }^{6)}$ recommended the use of a flap of the pulmonary artery wall with a corresponding flap from the aorta. Tension on the aortocoronary anastomosis is prevented and lengthening of the implanted vessel to bridge the distance between the anomalous left coronary artery and the aorta is provided, by this technique. Furthermore, extensive dissection of the anomalous coronary artery is not necessary with this technique, and by this way, bleeding complications and the distortion risk decreases. As viable native tissues are used in this procedure, the growth of the implanted artery with age is also provided.

Turley and colleagues described another technique for coronary lengthening. ${ }^{10)}$ In this technique, in order to bridge the gap between the anomalous coronary artery and the aorta, a noncircumferential segment of the pulmonary artery is used to provide a tunnel. As viable native tissues are used during this procedure, this technique also provides the future growth of the implanted artery. We used this technique in a few patients at our center with good early and late results. Detachment of the commissure between the posterior and the noncoronary sinuses was often necessary.

Direct transfer of the anomalous coronary artery and implantation into the aorta is the simplest way in the management of patients with anomalous origin of the left main coronary artery from the pulmonary artery and is the choice of treatment, if it is technically possible. This method has gained popularity through increased experience with coronary artery transfer techniques adapted from the arterial switch operation. The reported mortality rate is $0 \%$ to $16 \%{ }^{11)}$ Nonetheless, direct coronary transfer has some major limitations. There is a long distance between the coronary artery orifice and aorta in cases in which the anomalous coronary artery arises from the posterior pulmonary sinus close to the commissure between the posterior and the nonfacing sinus, or less frequently from the nonfacing sinus. Additionally, there is a tension in the aortocoronary anastomosis due to ventricular ischemia.

The management of mitral regurgitation at the time of anomalous origin of the left main coronary artery from the pulmonary artery repair varies between institutions and remains controversial. Mitral valve repair is not recommended by many surgeons suggesting anomalous origin of the left main coronary artery from the pulmonary artery surgery, with the improvement in coronary perfusion, ventricular dilation, and papillary muscle dysfunction, will also result in the improvement of mitral regurgitation. ${ }^{12,13)}$ However, some surgeons recommend the repair of severe mitral regurgitation at the time of the anomalous origin of the left main coronary artery from the pulmonary artery procedure, owing to the high incidence of persistent mitral regurgitation after coronary reimplantation.

We prefer to use aortic implantation of the anomalous 
artery by creating a tension-free composite tunnel made of aortic and pulmonary artery flaps. ${ }^{14)}$

In the surgical management of anomalous origin of the left main coronary artery from the pulmonary artery, regardless of the site of origin of the anomalous coronary artery, a dual-coronary system can be established in all patients. Composite tunnel implantation techniques allow tension-free anastomosis with minimal risk of distortion, acceptable operative mortality, excellent cardiac recovery, and late survival. Regardless of the implantation technique, the anomalous origin of the left main coronary artery from the pulmonary artery operation is associated with acceptable operative mortality, excellent cardiac recovery, and late survival.

\section{References}

1) Brooks HS. Two cases of an abnormal coronary artery of the heart arising from the pulmonary artery: with some remarks upon the effect of this anomaly in producing cirsoid dilatation of the vessels. J Anat Physiol 1885; 20: 26-9.

2) Keith JD. The anomalous origin of the left coronary artery from the pulmonary artery. Br Heart J 1959; 2: 149-61.

3) Kirklin JW, Barratt-Boyes BG. Cardiac surgery. John Wiley, New York, 1986.

4) Wesselhoeft H, Fawcett JS, Johnson AL. Anomalous origin of the left coronary artery from the pulmonary trunk. Its clinical spectrum, pathology, and pathophysiology, based on a review of 140 cases with seven further cases. Circulation 1968; 38: 403-25.

5) del Nido PJ, Duncan BW, Mayer JE Jr, et al. Left ventricular assist device improves survival in children with left ventricular dysfunction after repair of anoma- lous origin of the left coronary artery from the pulmonary artery. Ann Thorac Surg 1999; 67: 169-72.

6) Sese A, Imoto Y. New technique in the transfer of an anomalously originated left coronary artery to the aorta. Ann Thorac Surg 1992; 53: 527-9.

7) KITTLE CF, DIEHL AM, HEILBRUNN A. Anomalous left coronary artery arising from the pulmonary artery; report of a case and surgical consideration. J Pediatr 1955; 47: 198-206.

8) Dodge-Khatami A, Mavroudis C, Backer CL. Anomalous origin of the left coronary artery from the pulmonary artery: collective review of surgical therapy. Ann Thorac Surg 2002; 74: 946-55.

9) Kececioglu D, Voth E, Morguet A, et al. Myocardial ischemia and left-ventricular function after ligation of left coronary artery (Bland-White-Garland syndrome): a long-term follow-up. Thorac Cardiovasc Surg 1992; 40: 283-7.

10) Turley K, Szarnicki RJ, Flachsbart KD, et al. Aortic implantation is possible in all cases of anomalous origin of the left coronary artery from the pulmonary artery. Ann Thorac Surg 1995; 60: 84-9.

11) Takeuchi $S$, Imamura $H$, Katsumoto $K$, et al. New surgical method for repair of anomalous left coronary artery from pulmonary artery. J Thorac Cardiovasc Surg 1979; 78: 7-11.

12) Huddleston CB, Balzer DT, Mendeloff EN. Repair of anomalous left main coronary artery arising from the pulmonary artery in infants: long-term impact on the mitral valve. Ann Thorac Surg 2001; 71: 1985-8; discussion 1988-9.

13) Lange R, Vogt M, Horer J, et al. Long-term results of repair of anomalous origin of the left coronary artery from the pulmonary artery. Ann Thorac Surg 2007; 83: $1463-71$.

14) Murthy KS, Krishnanaik S, Mohanty SR, et al. A new repair for anomalous left coronary artery. Ann Thorac Surg 2001; 71: 1384-6. 